\title{
Tempo de reação e a eficiência do jogador de goalball na interceptação/defesa do lançamento/ataque
}

\author{
Reaction time and efficiency of the goalball player in interception/ \\ defense of throwing/attack
}

\author{
G.P. Silva, V.R. Pereira, P.P. Deprá, J.I. Gorla
}

\begin{abstract}
RESUMO
O goalball é um esporte coletivo criado para pessoas cegas e deficientes visuais. O objetivo do estudo foi analisar o comportamento motor de jogadores de goalball, de ambos os sexos, para avaliar a influência do tempo de reação (TRe) na eficiência das ações de defesa. Participaram do estudo nove atletas, que integraram as equipes nacionais de goalball durante os $3^{\circ}$ Jogos Mundiais da IBSA, realizados em São Caetano do Sul - SP, em Agosto de 2007. Para a coleta de dados utilizou-se a cinemetria, de modo a obter imagens das ações motoras dos sujeitos na realização das ações de defesa. Os dados foram analisados a partir da movimentação de segmentos corporais em relação à movimentação da bola, utilizando-se o programa de análise de movimento, o DgeeMe Version 1.0b. Constatou-se que os valores de TRe dos sujeitos estavam dentro dos padrões referenciados na literatura. A repetição dos movimentos e a prontidão para a ação influenciaram uma melhor resposta dos sujeitos em relação à tarefa, facilitando uma diminuição no TRe. Em relação às ações dos sujeitos que resultaram em defesa da bola ou em gol, não houve diferença de TRe entre os dois grupos de ações.

Palavras-chave: tempo de reação, deficiência visual, goalball
\end{abstract}

ABSTRACT

Goalball is a team sport designed for the blind and the visually impaired. This research aimed at analyzing the motor behavior of goalball players in order to evaluate the influence of reaction time (TR) in the efficiency of defense actions. Nine athletes from the national team of goalball, who played in the $3^{\text {rd }}$ World Games of IBSA (São Caetano do Sul/SP, August 2007), participated in this study. Data were collected through cinemetry in order to obtain images of motor movements during defense actions and then analyzed and developed using the biomechanics model of motor control of human movements, and treated using a software specific for analyzing movement, the DgeeMe Version 1.0b. Participants' TR met the standard mentioned in the literature. Repetition of movements and promptness to act influenced the quality of their responses and facilitated a decrease in TR. Keywords: reaction time, visual impairment, goalball

Submetido: 13.09.2009 | Aceite: 03.05.2010

Gilberto Pereira da Silva. Universidade Paranaense - Toledo/PR, Brasil.

Vanildo Rodrigues Pereira e Pedro Paulo Deprá. Universidade Estadual de Maringá - DEF - Maringá/PR, Brasil. José Irineu Gorla. Departamento de Estudos da Atividade Física Adaptada - FEF/UNICAMP/SP, Brasil.

Endereço para correspondência: Gilberto Carlos Pereira da Silva, Universidade Paranaense - UNIPAR Campus II,

Rua Santos Dumont, 2161, CEP 85.900-010, Toledo/Paraná, Brasil.

E-mail: gilbertosilva@unipar.br 
O goalball é uma modalidade esportiva coletiva, criada especialmente para jogadores cegos e deficientes visuais, cuja intervenção no jogo se dá principalmente através da mobilização das percepções tátil e auditiva.

Este esporte possui características comuns às outras modalidades coletivas e também particularidades que o distinguem das demais. Dentre elas destaca-se o uso de uma bola com guizos, para que os jogadores possam ter discriminação auditiva nas ações de jogo. Há necessidade do uso de vendas pelos jogadores, evitando possíveis vantagens dos deficientes visuais sobre os cegos e também há necessidade de silêncio absoluto no local do jogo.

Um fator crucial para a efetivação das ações de jogo é o tempo para realizá-las, pois entre a interceptação e o domínio da bola (defesa) e a ação do lançamento (ataque), o jogador não poderá exceder o tempo regulamentar de dez segundos, o que resultaria em uma penalidade. Assim, uma rápida análise das ações de jogo e sua relação com o tempo regulamentar para efetivar a jogada, permite inferir que esta modalidade é muito dinâmica, exigindo rapidez na discriminação da bola (identificação do estímulo), rapidez na tomada de decisão (seleção da resposta) e rapidez na ação de resposta (programação da resposta) em relação ao posicionamento de defesa e/ou nas ações de ataque.

Neste estudo optou-se por analisar o tempo de reação (TRe) dos jogadores de goalball, indicando a rapidez e a eficácia da tomada de decisão, que é uma medida de desempenho importante. Consiste, portanto, em estudar o comportamento motor dos jogadores frente ao jogo, uma vez que uma das funções desta área de estudo é questionar como os processos melhoram a eficácia e a eficiência da seleção e execução da resposta motora (Hoffman \& Harris, 2002). Para tanto, é necessário compreender, também, os mecanismos de controle motor, ao se analisar como os mecanismos de seleção e execução de resposta controlam os movimentos do corpo.
Assim, a presente investigação visa contribuir para o avanço no conhecimento sobre o controle motor dos cegos e deficientes visuais, nas ações motoras específicas deste esporte.

Problema semelhante foi observado por Tani (2005, p. 317) em relação aos portadores de deficiência visual:

“... considerando a importância do sistema visual no controle postural, seria possível especular que indivíduos portadores de deficiência visual não conseguiriam realizar atividades motoras com grande demanda de controle postural. Contudo, esses indivíduos não somente são capazes de realizar essas atividades, como demonstram ganhos significativos na capacidade de equilíbrio, quando submetidos a programas de intervenção".

Um estudo do comportamento motor de pessoas cegas e com deficiência visual, objetiva compreender como as habilidades motoras são aprendidas e controladas (Hoffman \& Harris, 2002) a fim de incrementar os métodos de intervenção junto a estes indivíduos.

Assim, o objetivo geral foi analisar o comportamento motor de jogadores de goalball cegos e deficientes visuais, de ambos os sexos, para avaliar a influência do tempo de reação na eficiência das ações de defesa.

Para operacionalizar o desenvolvimento da pesquisa, formularam-se os seguintes objetivos específicos: avaliar o tempo de reação e a eficiência de jogadores cegos e deficientes visuais na ação de interceptação/defesa do lançamento/ataque na modalidade goalball; verificar a existência de diferença para os tempos de reação entre jogadores de goalball do sexo masculino e feminino; verificar a existência de diferença para os tempos de reação entre as ações dos jogadores de goalball que resultaram em defesa da bola e as ações que resultaram em gol; revelar indicadores que venham a favorecer $o$ trabalho dos profissionais atuantes na área, quanto ao tempo de reação e a eficiência na resposta dos jogadores de goalball. 


\section{MÉTODO}

\section{Amostra}

Os participantes deste estudo foram 5 sujeitos do sexo masculino e 4 do sexo feminino, todos com cegueira e com deficiência visual, de ambos os sexos, que integraram a equipe brasileira de goalball que participou nos $3^{\circ}$ Jogos Mundiais da International Blind Sport Association (IBSA), realizado na cidade de São Caetano do Sul - SP, no mês de Agosto de 2007. Estes jogadores, por sua vez, foram convidados a participarem da Seleção Nacional após serem selecionados no Campeonato Brasileiro de Goalball.

A equipe masculina compreendeu atletas com idades entre 19 e 32 anos, sendo a média de idades de 26.20 anos ( \pm 5.80), com um tempo médio de prática na modalidade de 81.60 meses ( \pm 57.17 ), portanto, acima de 6 anos. Para a equipe feminina a idade variou entre 21 e 27 anos, com média de 23.50 anos ( \pm 3.00), com um tempo médio de prática da modalidade de 55.75 meses $( \pm 28.43$ ), girando em torno dos 4 anos.

Esta escolha se deu pelos resultados alcançados nos últimos Jogos Pan-Americanos da IBSA, realizados em São Paulo, em 2004, quando a equipe feminina conquistou o vicecampeonato e a equipe masculina o quarto lugar, demonstrando que os jogadores brasileiros apresentaram um ótimo nível técnico, constituindo-se, assim, em excelente fonte de pesquisa.

\section{Instrumentos e Procedimentos}

A pesquisa caracterizou-se como do tipo descritivo, envolvendo variáveis que buscaram descrever e estudar o tempo de reação e a eficiência dos avaliados que, é um estudo de status (Thomas \& Nelson, 2002), que neste caso busca avaliar os sujeitos executando uma determinada tarefa (Hoffman \& Harris, 2002) permitindo, no entanto, a comparação entre grupos semelhantes.

A coleta de dados dos atletas se deu no período de Julho de 2007, quando os voluntários participavam da segunda fase de treinamentos visando a participação nos $3^{\circ}$ Jogos Mundiais da IBSA.

Para a efetivação desta pesquisa foi solicitada permissão à Confederação Brasileira de Desportos para Cegos (CBDC), entidade responsável pela organização das seleções nacionais. Antes do início da avaliação, os participantes foram informados dos procedimentos da pesquisa e assinaram um termo de consentimento livre e esclarecido.

Foi solicitada, também, autorização para a realização do estudo junto ao Comitê Permanente de Ética em Pesquisa envolvendo Seres Humanos da Universidade Estadual de Maringá, sob o registro CAAE 0165.0.093.00007.

Para atender aos objetivos deste estudo, foi utilizado como instrumento a cinemetria, que é um método de avaliação cinemática que utiliza imagens filmadas da execução do movimento para observar o comportamento das variáveis dependentes, tais como posição do corpo e a duração do movimento. Estas são medidas de produção de desempenho, pois tem como base a gravação do movimento enquanto o sujeito está realizando a ação (Magill, 2000).

Para a obtenção das imagens na coleta de dados foi posicionada uma câmera filmadora A (figura 1), de modo a obter imagens das ações motoras dos voluntários na realização das ações de defesa.

A câmera de vídeo foi posicionada perpendicularmente ao plano sagital, hemicorpo direito do sujeito, de modo a filmar seus movimentos na ação de defesa da bola. Este posicionamento permitiu a avaliação do tempo de reação do voluntário quando da observação do lapso de tempo entre o lançamento da bola e a primeira ação realizada, permitindo também identificar qual parte do corpo realiza esta primeira ação.

As imagens foram registradas através de uma câmera de vídeo digital, com uma frequência de $30 \mathrm{~Hz}$, da marca SAMSUNG ${ }^{\circledR}$, modelo SC-D375 NTSC.

O momento do lançamento da bola foi identificado por um dispositivo luminoso 
(figura 1) que foi acionado por um auxiliar no exato momento em que a bola, ao ser lançada, entrava em contato com o solo.

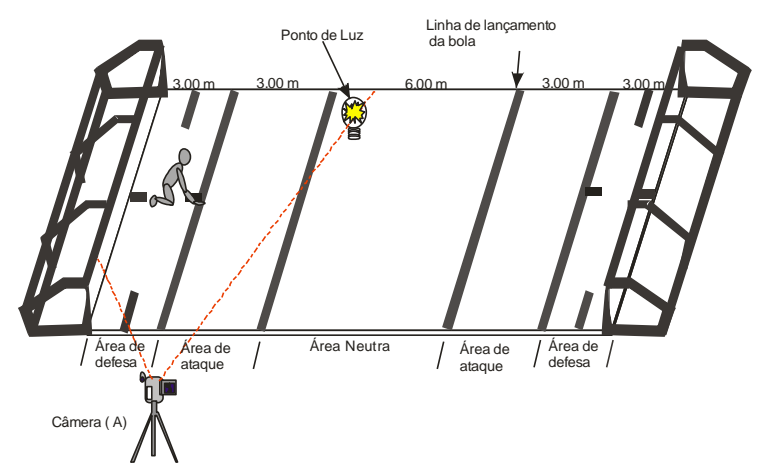

Figura 1. Posicionamento do sujeito avaliado e da câmera no local de filmagem

Antes do início da coleta de dados, foi permitido um aquecimento, que ocorreu a critério do voluntário do estudo, durante aproximadamente dez minutos. Após o período de preparação, foram posicionados sobre sua roupa marcadores auto-adesivos para auxiliar na avaliação dos movimentos. Foram posicionados marcadores nos seguintes processos anatômicos: (1) falange distal do quinto metatarso, (2) tornozelo, (3) joelho, (4) centro articular da articulação fêmur-quadril, (5) centro articular do ombro, (6) epicôndilo lateral do úmero, (7) processo estilóide da ulna, (8) eixo articular do quinto metacarpo, (9) queixo e (10) testa. Todos no lado direito do corpo.

Em seguida, foi concedido um período de três minutos de adaptação, onde o voluntário poderia praticar ações de defesa em condições semelhantes à do experimento. $\mathrm{O}$ voluntário deveria utilizar seu equipamento de jogo, principalmente a venda, equipamento fundamental para que o atleta participe de um jogo. Após o período de adaptação, o voluntário permaneceu em repouso por um período de dois minutos, no mínimo, a fim de evitar que alterações decorrentes do processo de fadiga interferissem no experimento.

O sujeito se posicionou imóvel na marcação central da área de equipe na quadra, podendo permanecer na sua posição habitual de defesa, e foi instruído a realizar ações de defesa com a finalidade de impedir que a bola lançada em direção à baliza se convertesse em gol.

A bola foi lançada próxima à linha que delimita a área de lançamento da quadra, no seu ponto médio. Foram realizados dez lançamentos de modo aleatório, e sem que o sujeito tivesse conhecimento prévio da direção dos lançamentos em cada posição. Após cada tentativa seria permitido que o sujeito se posicionasse na marca inicial.

$\mathrm{O}$ número de dez lançamentos refere-se à metade do número médio de ações de defesa realizados por jogadores de goalball de alto nível, na posição de 'central' (Mora, 1993), registrados para um jogo com dois tempos regulamentares de sete minutos. Deve-se ressaltar que quando este estudo foi realizado, o tempo regulamentar de jogo era de dois tempos de sete minutos, e atualmente é de dois tempos de dez minutos.

Para determinar os padrões de movimento que caracterizaram as ações de defesa dos voluntários e a consequente análise dos tempos de reação utilizados na realização da tarefa, foi obtido um conjunto de variáveis temporais a partir de uma análise dos movimentos do atleta. Com base na literatura (Magill, 2000), foi proposto um esquema do intervalo de tempo para as variáveis da pesquisa (figura 2 ).

O tempo de reação é o tempo que decorre entre a apresentação um estímulo nãoantecipado até o início da resposta da pessoa (Schmidt, 2001), assim, neste estudo, o tempo de reação do estímulo (TRe) representa o tempo decorrido entre o contato da bola no solo, quando do seu lançamento (sinal do estímulo), até a primeira ação realizada pelo sujeito para a retirada do apoio do solo ou troca do apoio (resposta) no sentido de realizar um movimento em direção à defesa da bola; foi chamado de tempo de movimento (TM) o tempo que o sujeito tem efetivamente para realizar a ação de defesa, ou seja, o tempo entre o início da resposta até o final da ação, portanto, o deslocamento do sujeito da posição 
inicial até o local em que julga adequado para realizar a defesa da bola; e, de tempo de resposta (TRES) o tempo total decorrido entre o momento do lançamento da bola (sinal do estímulo) até o contato do sujeito com a bola na ação da defesa (final da resposta) ou quando esta passa pela linha do corpo do sujeito quando não há a defesa da bola e consequentemente um gol é marcado. Foram observados também os lançamentos que resultaram em gol e os lançamentos onde houve a defesa da bola.

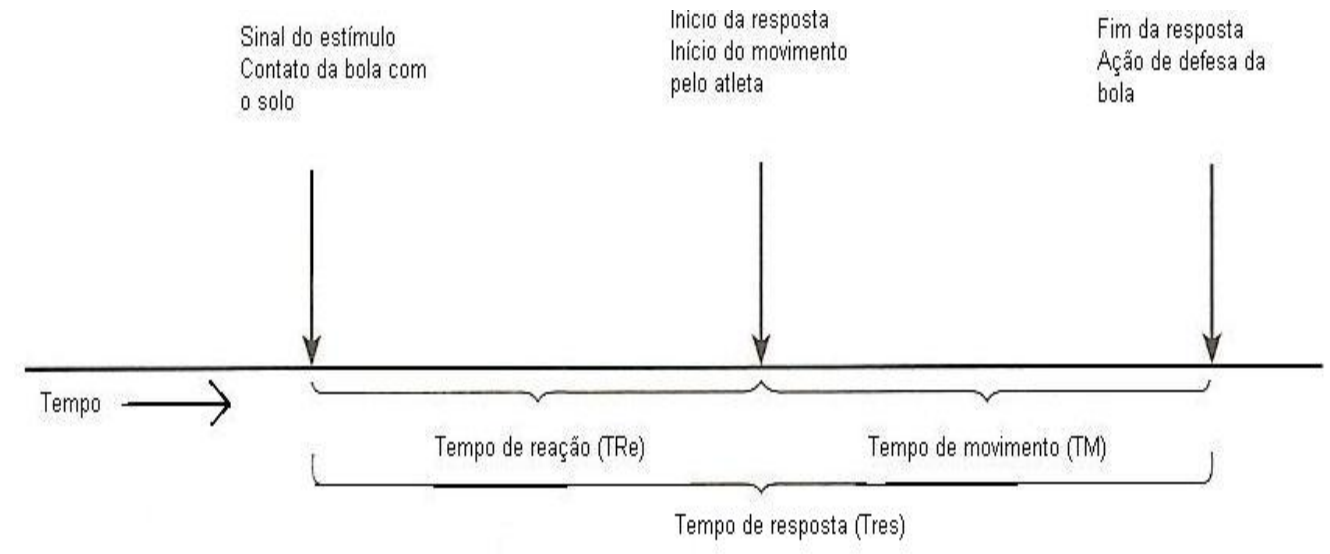

Figura 2. Esquema do intervalo de tempo das variáveis temporais

\section{Análise Estatística}

Para apresentação dos resultados foi utilizada estatística descritiva média e desvio padrão (DP). Também foi utilizado coeficiente de correlação linear de Pearson para determinar a relação entre as variáveis do estudo.

A normalização dos dados foi avaliada através do teste de Shapiro-Wilk. Como não foi encontrada normalidade para todas as variáveis do estudo, foi utilizado o teste de MannWhitney para comparação entre amostras independentes, para verificar a existência entre os resultados dos grupos masculino $\mathrm{e}$ feminino.

Os dados foram analisados através do pacote estatístico SPSS ${ }^{\circledR} 10.0$ para Windows ${ }^{\circledR}$.

\section{RESULTADOS}

Ao analisar as ações dos sujeitos, na tentativa de realizar a defesa da bola após o lançamento, foram encontrados dados que permitiram verificar o comportamento motor dos jogadores de goalball e avaliar a influência do tempo de reação sobre a eficiência das ações de defesa.
Constata-se, na tabela 1 , que as médias do grupo masculino para as variáveis tempo de reação (TRe) $121 \mathrm{~ms}$ ( \pm 88), tempo de movimento (TM) $662 \mathrm{~ms} \mathrm{(} \pm 233$ ), e tempo de resposta (TRES) $785 \mathrm{~ms}( \pm 216)$.

É importante salientar que esses tempos foram obtidos com imagens feitas com equipamentos de trinta hertz, ou seja, imagens filmadas a trinta quadros por segundo.

Tabela 1

Tempo médio (M) e desvio padrão (DP) das variáveis do estudo para os sujeitos do sexo masculino (tempo em milissegundos)

\begin{tabular}{ccccccc}
\hline \multirow{2}{*}{ Variáveis } & \multicolumn{2}{c}{ TRe $(m s)$} & \multicolumn{2}{c}{ TM $(m s)$} & \multicolumn{2}{c}{ TRES $(m s)$} \\
& $M$ & $D P$ & $M$ & $D P$ & $M$ & $D P$ \\
\hline Sujeito 1 & 201 & 76 & 576 & 253 & 777 & 254 \\
Sujeito 2 & 61 & 38 & 801 & 65 & 862 & 90 \\
Sujeito 3 & 171 & 77 & 431 & 129 & 610 & 176 \\
Sujeito 4 & 86 & 61 & 951 & 126 & 1037 & 124 \\
Sujeito 5 & 36 & 15 & 644 & 55 & 679 & 55 \\
Grupo & 121 & 88 & 662 & 233 & 785 & 216 \\
\hline
\end{tabular}

TRe: tempo de reação; TM: tempo de movimento; TRES: tempo de resposta 
Relacionando os dados observados, foram encontradas duas correlações significativas (tabela 2): i) entre o tempo de resposta (TRES) e o tempo de movimento (TM), com um $r$ de .92, o que representa uma correlação muito forte entre os dados apresentados; e, ii) entre o tempo de reação (TRe) e o tempo de movimento (TM) com um $r$ de -.35 , apresentando uma fraca correlação negativa entre as variáveis. Salienta-se que, embora esta correlação seja fraca, ainda assim pode-se dizer que ela existe (Tritschler, 2003).

Tabela 2

Coeficientes de correlação simples (r) entre as variáveis do grupo masculino ( $n$ de lançamentos $=42$ )

\begin{tabular}{crl}
\hline Variáveis & $r$ & $p$ \\
\hline TRES - TM & .92 & .01 \\
TRe - TM & -.35 & .05 \\
TRe - TRES & .37 & - \\
\hline
\end{tabular}

TRe: tempo de reação, TM: tempo de movimento, TRES: tempo de resposta

A tabela 3 apresenta os valores da média (M) e do desvio padrão (DP) para as variáveis TRe, TM e TRES para os sujeitos do sexo feminino. Constatou-se que as médias do grupo feminino para as variáveis foram: tempo de reação (TRe) $152 \mathrm{~ms}$ ( \pm 101 ), tempo de movimento (TM) $901 \mathrm{~ms}$ ( \pm 180 ) e o tempo de resposta (TRES) $1053 \mathrm{~ms}( \pm 157)$.

Tabela 3

Tempo médio (M) e desvio padrão (DP) das variáveis do estudo para os sujeitos do sexo feminino (tempo em milissegundos)

\begin{tabular}{ccccccc}
\hline \multirow{2}{*}{ Variáveis } & \multicolumn{2}{c}{ TRe $(m s)$} & \multicolumn{2}{c}{ TM $(m s)$} & \multicolumn{2}{c}{ TRES $(m s)$} \\
& $M$ & $D P$ & $M$ & $D P$ & $M$ & $D P$ \\
\hline Sujeito 1 & 92 & 43 & 1016 & 313 & 1108 & 294 \\
Sujeito 2 & 193 & 63 & 873 & 85 & 1068 & 104 \\
Sujeito 3 & 70 & 63 & 935 & 88 & 1005 & 169 \\
Sujeito 4 & 212 & 115 & 832 & 168 & 1044 & 127 \\
Grupo & 152 & 101 & 901 & 180 & 1053 & 157 \\
\hline
\end{tabular}

TRe: tempo de reação; TM: tempo de movimento; TRES: tempo de resposta

Relacionando os dados observados para o grupo feminino, também foram encontradas duas correlações, que são apresentadas na tabela 4: i) entre o tempo de resposta (TRES) e o tempo de movimento (TM) com um $r$ de .83, o que representa haver uma forte correlação entre estas variáveis da pesquisa; ii) entre o tempo de reação (TRe) e o tempo de movimento (TM) encontrou-se um $r$ de -.49 , o que representa uma fraca correlação negativa (Tritschler, 2003).

Tabela 4

Coeficientes de correlação simples (r) entre as variáveis do grupo feminino ( $n$ de lançamentos $=26$ )

\begin{tabular}{ccc}
\hline Variáveis & $r$ & $p$ \\
\hline TRES - TM & .83 & .01 \\
TRe - TM & -.49 & .05 \\
TRe - TRES & .07 & - \\
\hline
\end{tabular}

TRe: tempo de reação, TM: tempo de movimento, TRES: tempo de resposta

Para verificar se houve diferença significativa entre as médias do grupo masculino e feminino para as variáveis estudadas, foi utilizado o teste de MannWhitney, cujo resultado, apresentado na tabela 5 , revelou que o resultado encontrado para as variáveis TRe e TM não apresentou diferenças significativas ao nível de $p<.05$. Assim, podese afirmar que não há diferenças significativas entre as médias dos dois grupos.

Tabela 5

Diferença entre as médias das variáveis para os grupos masculino e feminino

\begin{tabular}{ccc}
\hline & Masculino & Feminino \\
\hline$n$ & 42 & 26 \\
TRe & \multicolumn{1}{c}{-1.321} \\
TM & -4.468 \\
TRES & \multicolumn{2}{c}{$-5.494^{*}$} \\
\hline${ }^{*} p<.001 ;$ TRe: tempo de reação, TM: tempo de \\
movimento, TRES: tempo de resposta
\end{tabular}

No entanto, para a variável TRES, os resultados demonstraram que existem diferenças significativas entre as médias desta variável para os grupos masculino e feminino (Tritschler, 2003). Portanto, o grupo masculino apresenta um TRES mais rápido que o grupo feminino. 
Outro aspecto considerado nesta pesquisa foi a verificação da existência de diferenças entre as ações dos sujeitos para os lançamentos que resultaram em gol e os lançamentos onde houve a defesa da bola. A tabela 6 apresenta os resultados encontrados, onde se observou, para as ações que resultaram em gol, um TRe de $139 \mathrm{~ms}( \pm 75)$, TM de $725 \mathrm{~ms}$ ( \pm 231 ), e um TRES de $866 \mathrm{~ms}$ ( \pm 240 ). Já para as ações que resultaram em defesa da bola, foram obtidos os seguintes valores: TRe de $128 \mathrm{~ms}$ ( \pm 106 ), um TM de $773 \mathrm{~ms}$ ( \pm 251 ), e um TRES de $902 \mathrm{~ms}$ ( \pm 231$)$.

\section{Tabela 6}

Tempo médio (M) e desvio padrão (DP) das variáveis do estudo para os lançamentos convertidos em gol e os lançamentos em que ocorreram defesas (tempo em milissegundos)

\begin{tabular}{ccccccc}
\hline Variáveis & \multicolumn{2}{c}{ TRe $(m s)$} & \multicolumn{2}{c}{ TM $(m s)$} & \multicolumn{2}{c}{ TRES $(m s)$} \\
& $M$ & $D P$ & $M$ & $D P$ & $M$ & $D P$ \\
\hline $\begin{array}{c}\text { Defesa } \\
(n=39) \\
\quad\end{array}$ & 128 & 106 & 773 & 251 & 902 & 231 \\
$\begin{array}{c}\text { Gol } \\
(n=29)\end{array}$ & 139 & 75 & 725 & 231 & 866 & 240 \\
\hline
\end{tabular}

TRe: tempo de reação; TM: tempo de movimento; TRES: tempo de resposta

Para verificar se houve diferença significativa entre as médias dos lançamentos convertidos em gol e os lançamentos em que os sujeitos conseguiram realizar a defesa da bola, utilizou-se o teste de Mann-Whitney.

Tabela 7

Diferença entre as médias das variáveis para as ações que resultaram em gol e as ações que resultaram em defesa da bola

\begin{tabular}{ccrc}
\hline & Defesa & Gol \\
\hline$n$ & 39 & & 29 \\
TRe & & .454 & \\
TM & & -.810 & \\
TRES & & .609 & \\
\hline
\end{tabular}

TRe: tempo de reação, TM: tempo de movimento, TRES: tempo de resposta

Os resultados apresentados na tabela 7 demonstram que o valor obtido foi menor do que o valor crítico para todas as variáveis.
Assim, pode-se afirmar que as médias das variáveis não apresentaram diferenças significativas ao nível de $p<.05$, e, portanto foram semelhantes.

\section{DISCUSSÃO}

O foco central do presente estudo está na natureza da informação sobre o comportamento motor de jogadores de goalball, de ambos os sexos, na tentativa de avaliar a influência do tempo de reação na eficiência de suas ações de defesa. É através do comportamento motor que se pretende compreender as variáveis que determinam a eficácia da execução motora e da aprendizagem sobre a execução ou conduta motora específica (Schimdt, 2001).

Um estudo realizado para analisar o desempenho humano (Araújo, 2002) propõe que um estímulo externo para iniciar uma ação se inicia com o processo de identificação do estímulo, seguido da seleção da resposta e da programação da resposta, chegando finalmente à evocação dos comandos de movimento para os músculos. Assim, o estágio de identificação da resposta começa com a percepção do sinal do estímulo vindo do ambiente, observando que quanto mais intenso e discriminado o estímulo, mais rapidamente a pessoa poderá responder a ele.

Sendo o tempo de reação o intervalo de tempo que decorre entre um estímulo nãoantecipado e o início da resposta da pessoa, representa também o tempo que esta leva para tomar uma decisão e iniciar uma ação como reposta ao estímulo, indicando a velocidade de processamento da informação (Schimdt, 2001). Partindo desta premissa, buscou-se junto a um grupo de elite no desporto para deficientes visuais, notadamente os atletas da seleção brasileira de goalball, informações que explicassem a velocidade da resposta motora dos sujeitos cegos e deficientes visuais frente a esta situação experimental.

$\mathrm{Na}$ execução da coleta de dados, os lançamentos foram executados por diferentes sujeitos. Como os resultados apresentaram 
semelhanças, foram tratados em conjunto, sendo diferenciados apenas por sexo.

O grupo masculino obteve uma média de tempo de reação (TRe) de $121 \mathrm{~ms}( \pm 88$ ), portanto, dentro dos parâmetros de tempo de reação ao sinal acústico apresentado por Zaciorskij (cit. por Weineck, 2005) com duração em torno de 120 a 270 ms, apresentado como padrão para pessoas que estão na média da população, podendo atletas realizarem tempos mais rápidos, em torno de 50 a 160 ms (Zakharov, 1992).

Com base nas propostas de Jongsma e colaboradores, Teixeira (2006) indica o tempo de reação auditivo de aproximadamente 150 $\mathrm{ms}$ como valor de referência. $\mathrm{O}$ grupo feminino revelou dados semelhantes, com uma média de tempo de reação de $152 \mathrm{~ms}$, portanto, também dentro dos valores citados. Assim, podem-se considerar dois aspectos como intervenientes na obtenção desses valores pelos sujeitos: a repetição do movimento e a prontidão do sujeito para a ação.

A repetição do movimento é uma característica muito conhecida do desempenho humano, tendo-se observado que, quando da realização das ações de defesa pelos sujeitos em uma sequência de execução, o tempo de reação na tentativa seguinte será menor do que na tentativa anterior (Magill, 2000). Tal fato pode ter ocorrido, uma vez que o número de dez repetições de ações de defesa realizadas pelo sujeito parece não ser suficiente para induzi-lo à fadiga e interferir na performance do tempo de reação da tarefa.

Com relação à prontidão do sujeito, Magill (2000) relata evidências de que o tempo de reação é significativamente mais rápido quando o estímulo a ser respondido é precedido por um sinal de alerta. Isto de fato ocorre na modalidade goalball, uma vez que a bola, ao ser movimentada pelo lançador, em situação de deslocamento na quadra ou na preparação do lançamento, emite sons. Assim, o sujeito que atua na defesa pode discriminar a posição inicial da bola e preparar-se para defendê-la o mais rápido possível, diminuindo o tempo de reação.

Os testes de correlação intra-grupo revelaram resultados semelhantes, com uma forte correlação entre o tempo de resposta (TRES) e o tempo de movimento (TM), com $r$ de $.92(p<.01)$ para o grupo masculino e de $.83(p<.01)$ para o grupo feminino, demonstrando que, com o aumento do tempo de movimento, também aumenta o tempo de resposta. Acredita-se que, provavelmente, a velocidade com que o sujeito executa o movimento em direção à defesa está relacionada à velocidade da bola. Assim, se ele percebe que a velocidade de deslocamento da bola é menor, também poderá se movimentar com menor rapidez em direção à bola.

Para as variáveis tempo de reação (TRe) e tempo de movimento (TM), as correlações encontradas nos grupos masculino $(-.35, p<$ $.05)$ e feminino $(-.49, p<.05)$ são consideradas fracas, pois estas variáveis são medidas algo independentes, o que significa que não se pode utilizar o TRe para prever o TM e vice-versa (Magill, 2000). Entretanto, observou-se na presente pesquisa uma associação significativa entre essas variáveis.

Existe evidência na literatura de que não faz sentido a comparação entre TMs para diferentes tarefas (Teixeira, 2006), entretanto a comparação desta medida entre variações de uma mesma tarefa fornece informações sobre o modo pelo qual as ações são controladas.

Buscando verificar a existência de diferença para os tempos de reação entre os sujeitos dos grupos masculino e feminino, considerando as variáveis do estudo e os dados apresentados, foi encontrado um valor médio das variáveis do grupo masculino menor em relação ao grupo feminino, da ordem de $25.62 \%$ para o TRe, $36.10 \%$ para o TM e $34.14 \%$ para o TRES.

Estes valores indicam que o grupo masculino apresentou um tempo de reação (TRe) $25.62 \%$ menor que o grupo feminino, fato que pode ser explicado se for considerado que o grupo masculino possui uma média de tempo de prática da modalidade superior em 
dois anos ao grupo feminino. Consequentemente, está a mais tempo realizando ações de jogo e vivenciando situações em que o tempo de reação é fundamental para a execução de determinada técnica, seja em situação de estímulo simples ou complexo (Zakharov, 1992). Para o tempo de movimento (TM), o percentual de diferença foi de $36.10 \%$, demonstrando que o tempo para a realização da ação de defesa pelo grupo feminino foi maior, provavelmente em função da menor velocidade com que bola percorria o espaço da quadra desde o lançamento até o momento da defesa.

O tempo de resposta (TRES) foi $34.14 \%$ menor no grupo masculino, o que revela que a velocidade dos lançamentos é maior neste grupo. Isto se deve provavelmente em consequência da menor potência muscular da mulher em relação ao homem, e também pelo fato da bola ter um tamanho único, o que a torna mais difícil de ser manuseada pela mulher no momento da efetivação do lançamento, uma vez que sua mão também é usualmente menor.

Entretanto, na análise de comparação entre as médias das variáveis para os grupos masculino e feminino, verificou-se nos resultados para a variável TRES, que os dados indicaram a existência de diferença significativa $(p<.05)$ entre as médias destas variáveis, com valores de -5.494 , respectivamente, e um $p<$ .001 (Tritschler, 2003). Assim, pode-se afirmar que o grupo masculino obteve um TRES mais rápido que o grupo feminino. Para as variáveis TM e TRe não foi encontrada qualquer diferença significativa, portanto, pode-se afirmar que as médias do tempo de reação para os grupos masculino e feminino foram semelhantes.

Outro aspecto analisado na pesquisa foi a verificação da existência de diferenças entre as ações dos sujeitos que resultaram em defesa da bola $(n=39)$ e as que resultaram em gol ( $n=$ 29). Os resultados revelaram que, para as ações que resultaram na defesa da bola (128 \pm $106 \mathrm{~ms}$ ), obteve-se um TRe $8.59 \%$ menor do que nas ações que resultaram em gol (139 \pm $75 \mathrm{~ms})$. Embora o TRe apresente uma diferença pequena, pode-se afirmar que nas ações que resultaram na defesa da bola os sujeitos reagiram mais rápido e com mais eficiência na ação de defesa.

O tempo de movimento (TM) das ações que resultaram em gol $(725 \pm 231 \mathrm{~ms})$ foi $6.62 \%$ mais rápido do que as ações que resultaram em defesa (773 $\pm 251 \mathrm{~ms})$, demonstrando que nas ações que resultaram em gol, os sujeitos tiveram um tempo menor para realizar o movimento de defesa, em função da maior rapidez do deslocamento da bola.

$\mathrm{Na}$ comparação das médias entre as duas ações, de gol e de defesa, os resultados indicam que as médias das variáveis não apresentam diferenças significativas, podendo-se afirmar que as médias foram semelhantes.

Portanto, os indicadores encontrados, com todos os resultados das diferentes variáveis de reação e eficiência face à ação do opositor, da velocidade e direção da bola, poderão orientar a atividade de ensino e de treino dos profissionais da área, podendo influir, inclusive, na determinação do estilo e da técnica de ação a serem executados pelos atletas.

No momento do planejamento do treino, o treinador deve considerar a necessidade da utilização de exercícios de repetição com o objetivo de melhorar a resposta motora do atleta, diminuindo o tempo de movimento necessário à defesa da bola, bem como variar as formas de execução dos lançamentos a fim de criar novos estímulos a serem vivenciados pelos atletas.

Como o tempo de reação é dependente do tempo de percepção do sinal do estímulo, somado ao tempo de condução nervosa, mais o tempo de processamento e o tempo de resposta, este se apresenta como uma capacidade motora pouco treinável. No entanto, o treinamento das ações de jogo deve estar voltado não para a melhoria específica do tempo de reação, mas para mantê-lo nos melhores níveis possíveis. Os atletas deverão 
desenvolver uma maior capacidade de discriminação do sinal de estímulo, utilizando as dicas do ambiente para auxiliá-lo na diminuição de incertezas no estágio de seleção de respostas, na redução do tempo de programação dos movimentos, o que aumentaria a eficiência do desempenho.

Em relação ao tempo de reação entre as ações dos sujeitos que resultaram em defesa da bola e as ações que resultaram em gol, verificou-se que não há diferença entre os grupos de ações. Assim, entende-se que nas ações que resultaram em gol, os sujeitos provavelmente não estavam posicionados no local adequado para realizar a defesa da bola ou não se movimentaram com a velocidade necessária para realizar tal posicionamento. Portanto, entende-se que há a necessidade de implementar um treinamento voltado para a melhoria da técnica de defesa e, principalmente, para a velocidade de execução do movimento, a fim de permitir uma defesa eficiente.

Deve-se salientar que alguns fatores influenciaram de modo a criar limitações para este estudo. Basicamente, os dados das variáveis foram coletados de imagens feitas com equipamentos de trinta hertz, sendo as imagens filmadas a trinta quadros por segundo. Entretanto, entende-se que seria mais adequado o uso de um equipamento de gravação de alta velocidade o que tornaria os resultados mais precisos. Outro fator limitante do estudo foi o pequeno número de sujeitos avaliados, não permitindo generalizar os resultados encontrados. No entanto, buscou-se avaliar sujeitos que atuam nas seleções nacionais, masculina e feminina, na tentativa de apresentar dados de atletas de alto nível, que possam ser utilizados como indicadores de performance pelos treinadores, com a finalidade de otimizar seus treinamentos e melhorar a performance de suas equipes.
Ao se avaliar o tempo de reação e a eficiência dos sujeitos na ação de interceptação/defesa do lançamento/ataque na modalidade goalball, verificou-se que os valores do tempo de reação (TRE) dos sujeitos, para ambos os sexos, estão dentro dos padrões apresentados pelos pesquisadores referenciados na discussão deste estudo. Eles apontam, ainda, que a repetição dos movimentos e a prontidão para a ação são fatores que influenciam uma melhor resposta dos sujeitos em relação à tarefa, facilitando uma diminuição no tempo de reação (TRE). Os indicadores encontrados poderão orientar as atividades de ensino e de treinamento nesta área de atuação, particularmente no âmbito do goalball.

\section{REFERÊNCIAS}

Araújo, D. (2002). Tomada de decisão no desporto. Lisboa: Edições FMH.

Hoffman, S., \& Harris, J. C. (2002). Cinesiologia: O estudo da atividade física. Porto Alegre - RS: Artmed Editora.

Magill, R. A. (2000). Aprendizagem motora: Conceitos e aplicações. São Paulo - SP: Edgard Blücher, Ltda.

Mora, D. H. (1993). Analisis i planificacion de la temporada de l'equip de Barcelona de goalball. Barcelona: INEF.

Schmidt, R. A. (2001). Aprendizagem e performance motora: Uma abordagem da aprendizagem baseada no problema ( $2^{\mathrm{a}}$ ed.). Porto Alegre - RS: Artmed Editora.

Tani, G. (2005). Comportamento motor: Aprendizagem e desenvolvimento. Rio de Janeiro - RJ: Guanabara Koogan.

Teixeira, L. A. (2006). Controle motor. Barueri - SP: Manole.

Thomas, J. R., \& Nelson, J. K. (2002). Métodos de pesquisa em atividade física ( $3^{\mathrm{a}} \mathrm{ed}$.). Porto Alegre - RS: Artmed Editora.

Tritschler, K. A. (2003). Medida e avaliação em educação física e desportos de Barrow e McGee. Barueri-SP: Manole.

Weineck, J. (2005). Biologia do esporte ( $7^{\mathrm{a}}$ ed.). Barueri - SP: Manole.

Zakharov, A. (1992). Ciência do treinamento desportivo. Rio de Janeiro: Palestra Sport. 